\title{
Far-infrared modulated photoluminescence spectroscopy of InSb/GaSb quantum dot structures
}

\author{
R. A. Child, R. J. Nicholas, N. J. Mason, and P. A. Shields \\ Department of Physics, Oxford University, Clarendon Laboratory, Parks Road, Oxford OX1 3PU, United Kingdom \\ J.-P. R. Wells, I. V. Bradley, and J. Phillips \\ FOM Institute Rijnhizen, P.O. Box 1207, NL-3430BE Nieuwegein, The Netherlands \\ B. N. Murdin \\ Department of Physics, University of Surrey, Guildford GU2 7HX, United Kingdom
}

(Received 11 March 2003; revised manuscript received 9 July 2003; published 13 October 2003)

\begin{abstract}
The first far-infrared modulated photoluminescence (FIRM-PL) measurements in InSb/GaSb quantum dots have been performed. Far-infrared absorption is found to both enhance and suppress the quantum dot PL depending on the FIR intensity. This behavior is attributed to the nonthermal distribution of carriers amongst the quantum dots. The spectral dependence of the FIRM-PL signal measures the energy spectrum of the quantum dots, showing a peak at $14.5 \mathrm{meV}$ corresponding to transitions between the first two energy levels of the dot distribution.
\end{abstract}

DOI: 10.1103/PhysRevB.68.165307

PACS number(s): 78.67.Hc, 73.21.La, 78.30.Fs

\section{INTRODUCTION}

The study of quantum dots in semiconductors has proved highly fruitful recently, with the majority of work concentrating on the properties of the InAs/GaAs system. Recently it has been found that by the use of $\mathrm{InSb} / \mathrm{GaSb}$ structures it is possible to extend the emission wavelengths to the region around $2 \mu \mathrm{m} .{ }^{1,2}$ As a result of the much shallower confinement potential, these structures were shown to have substantially smaller confinement energies. In this work we report on a study of the quantum dot levels in this system by using direct intradot transitions detected using the doubleresonance technique of far-infrared modulated photoluminescence $^{3}$ (FIRM-PL). This enables us to measure electron states independently and to demonstrate the existence of excited states as well as giving an improved resolution for the quantum dot energy levels compared to interband measurements.

There have been several measurements of direct absorption intraband spectroscopy in semiconductor quantum dots, ${ }^{4-8}$ however these usually require multiple electron occupancy of the dots induced by either doping or high interband optical excitation intensity. ${ }^{9}$ In this work we use the FIRM-PL technique in which the use of a tunable high power FIR laser enables the average dot occupancy to be relatively low. This technique has previously been used by Murdin et al. ${ }^{10}$ to study InAs/GaAs quantum dots allowing them to make an unequivocal assignment of the resonant intradot transitions simultaneously with the interband excitation in neutral dots.

The InSb/GaSb dot system has a number of substantial differences from most other semiconductor dot systems, due to the relatively shallow dot confinement potential. This leads to much stronger "lateral communication" than is thought to occur in most other quantum dot systems. ${ }^{2}$ In contrast to InAs/GaAs quantum dots, where the distribution of occupied dots is frozen-in at more than $100 \mathrm{~K}$, the energy of the wetting layer is close to that of the dots. It is believed that the excitons remain in thermal equilibrium until quite low temperatures and hence populate more of the lowest energy dots in the overall distribution of dot sizes and energies. A further important question is whether or not the confinement is strong enough for the dots in this system to contain excited states, since none could be resolved in the previous PL studies. ${ }^{2}$

\section{EXPERIMENT}

The samples studied consisted of $\mathrm{InSb} / \mathrm{GaSb}$ selfassembled quantum dots grown on both GaAs [sample $A$ (3182)] and GaSb [sample $B$ (4174)] substrates by metal organic vapor phase epitaxy. Sample $A$, with the GaAs substrate material is the same sample as used in Ref. 2, where a $2-\mu \mathrm{m}$ buffer layer of GaSb was first grown, followed by a low-temperature 4-s layer of InSb and a capping layer of $\mathrm{GaSb}$. In the case of the GaSb substrate it was only necessary to grow a thin, $\sim 0.5-\mu \mathrm{m}$ buffer layer before a similar dot growth sequence. The buffer layer is $p$-type with a doping level of $\sim 10^{22} \mathrm{~m}^{-3}$, leading to some equilibrium hole population in the dots. The dot density is estimated to be $\sim 10^{13} \mathrm{~m}^{-2}$. The dot dimensions were typically found by atomic force microscopy (AFM) to be $\sim 38 \mathrm{~nm}$ in diameter and about 4-nm high. ${ }^{1}$

The experiments were performed at FELIX, the Free Electron Laser (FEL) facility at FOM, Rijnhuizen, which is continuously tunable from $\sim 4.5$ to $250 \mu \mathrm{m}$. The FIR comes in macropulses $5-\mu$ s long, each separated by $200 \mathrm{~ms}$, and each macropulse consists of a train of micropulses about $4 \mathrm{ps}$ in duration with a separation of $1 \mathrm{~ns}$. The experiments were performed by measuring the basic PL signal simultaneously with the FIR modulated component while scanning a monochromator. The FIR energy was then changed and the spectra recorded again. The sample was mounted on the cold finger of a continuous flow liquid-helium cryostat with polypropylene windows to transmit the FIR light. A $0.4 \mathrm{~W} \mathrm{~cm}^{-2} \mathrm{cw}$ $\mathrm{HeNe}$ laser was used to excite the interband transition, incident on the top surface of the sample. The photolumines- 


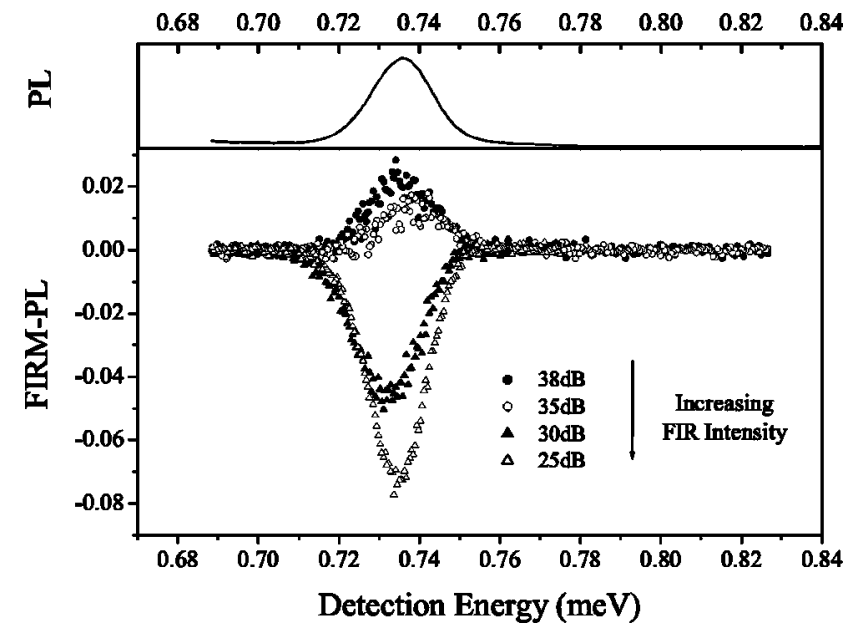

FIG. 1. The FIRM-PL signal of sample $A$ as a function of photon energy for different FIR attenuation levels (in $\mathrm{dB}$ ) at a FIR wavelength of $90 \mu \mathrm{m}$.

cence was collected and focused into a Triax $320300 \mathrm{~g} / \mathrm{mm}$ grating monochromator and detected by a liquid nitrogen cooled Ge $p-i-n$ diode detector. FELIX was used as the FIR source, illuminating the back of the sample through a hole in the mounting plate, inducing a change in the photoluminescence. The detector response time was sufficient to detect the overall modulation at $5 \mathrm{~Hz}$ produced by the macropulses, but was too slow to resolve the individual micropulses. The Ge detector had a dc output and an ac output enabling the simultaneous collection of the photoluminescence and FIRM-PL signal.

\section{FIRM-PL}

The energy region chosen for study, between 5 and 25 $\mathrm{meV}$, is readily available using FELIX and was based on the confinement energies of the dots which are known to lie in the range of $6-20 \mathrm{meV}^{2}$

\section{A. FIR power dependence}

Before the frequency dependence was studied on sample $B$ (4174), a study was first performed on the FIR power dependence at $4.2 \mathrm{~K}$ on sample $A$ (3182) which has been studied extensively by Alphandéry et al. ${ }^{1,2}$ In this sample the PL signal is dominated by the quantum dot signal at $0.73 \mathrm{eV}$ as shown in Fig. 1.

The FIR power dependence of the FIRM-PL signal is also illustrated in Fig. 1 using a FIR energy of $14 \mathrm{meV}$. It shows that the intensity level of the FIR can have a significant effect on the modulated photoluminescence signal with two regimes being observed. Very low intensity FIR was found to enhance the dot photoluminescence, while higher FIR intensities caused the PL to be suppressed, leading to a negative FIRM-PL signal. The intensity at which the response changed sign was also energy dependent, causing distortions in the line shape at intermediate power levels. At the lowest FIR intensities the overall FIRM-PL signal is biased slightly towards lower energy. As the intensity increases the low- energy side of the resonance is the first to change sign, causing an apparent shift to higher energies in the $35 \mathrm{~dB}$ trace. By $30 \mathrm{~dB}$ the high-energy side of the resonance is passing through zero and the peak appears to be shifted to lower energies and by $25 \mathrm{~dB}$ the whole peak is giving a negative FIRM-PL response.

This behavior can be understood through consideration of the redistribution of carriers amongst dots with a distribution of dot energies. In the absence of the FIR, at low temperatures the distribution of the photoexcited electrons is nonthermal as they at first cool and then become localized within a particular subset of quantum dots. This occurs once they are sufficiently strongly bound so that there is insufficient time for the electrons to thermalize within the distribution of dot energies before recombination happens. By contrast the much larger density of equilibrium holes coming from the p-type GaSb matrix are primarily localized in the larger dots. In InSb quantum dots, the transition to a nonthermal distribution was shown to occur at $\sim 10 \mathrm{~K}$ which is a factor of order 10 lower in temperature than for the InAs/GaAs system due to the much smaller localization energy. ${ }^{11}$ The resulting dot occupancy corresponds to a distribution whose average lateral confinement energy is $\sim 6 \mathrm{meV}^{2}$

When the sample is irradiated with low intensity FIR energy the carrier distribution can be strongly influenced as electrons within individual dots will be excited to higher energies where they have a higher escape rate due to the reduced localization energy and the possibility of further FIR absorption. This provides a mechanism whereby the carriers can show enhanced communication and thus increased PL efficiency, as shown schematically in Fig. 2(a). The shorter electron escape time compared with the recombination time enables the preferential population of larger, lower-energy dots to occur and also increases the probability of exciton formation. This causes an increase in PL intensity and the increase in the population of larger, lower-energy dots results in the FIRM-PL energy shifting slightly to lower energies.

Beyond a certain level of FIR intensity, which will be referred to as the FIR high power limit, gross heating of the carriers will occur. For the sample studied here this occurred at around an attenuation level of $32 \mathrm{~dB}$. A typical initial beam intensity of $20 \mathrm{~mW}$ with an additional attenuation of $30 \mathrm{~dB}$ is used to illuminate an area of $\sim 2 \mathrm{~mm}^{2}$. This gives a mean power density per micropulse of $5 \mathrm{~kW} \mathrm{~cm}^{-2}$. The power density during a macropulse is $20 \mathrm{~W} \mathrm{~cm}^{2}$. This is a considerable amount of power and could result in significant heating of the carriers, which we estimate by comparison with the temperature-dependent PL study of Alphandéry et $a .^{2}{ }^{2}$ to generate temperatures approaching $40 \mathrm{~K}$. Both electrons and holes are redistributed back to higher-energy dots with higher confinement energies and smaller localization energies. This results in reduced levels of dot photoluminescence as the electrons can leave the dots and spend more time in the wetting layer and the bulk material, enhancing the competing recombination routes as shown schematically in Fig. 2(b). In the case of the GaAs substrate sample $A$ where nonradiative recombination is dominant this leads to an overall decrease in the PL signal, whereas for sample $B$ with the $\mathrm{GaSb}$ substrate, there is a corresponding increase in the re- 

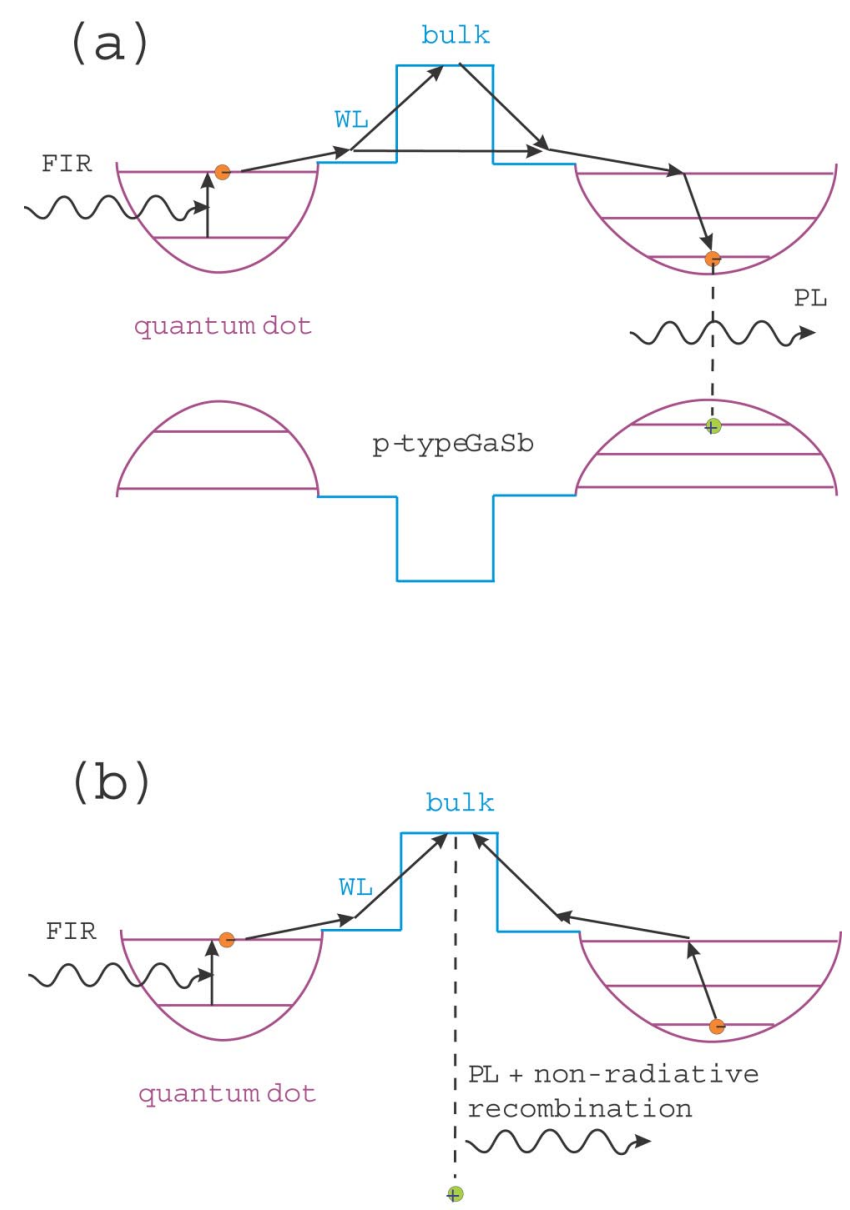

FIG. 2. A schematic depiction of the processes which influence the FIRM-PL signal in the (a) low-temperature, low FIR intensity limits, and (b) in the high-temperature/high FIR intensity limits. Two quantum dots of different sizes are shown schematically to simulate the distribution of quantum dot confinement energies.

sponse from the WL and substrate peaks as shown in Fig. 3. This would also lead to an increase in the mean energy of the occupied dots, biasing the FIRM-PL towards higher energy. This is thought to be the explanation for the persistence of the positive FIRM-PL signal up to slightly higher FIR intensity levels.

\section{B. Frequency dependence}

In order to study the spectral response of the FIRM-PL a series of experiments was performed using sample $B$ in which the FIR energy was swept in order to study the quantum dot energy levels. The disadvantage of sweeping FELIX, particularly over a large bandwidth, is that over time the power may vary. This means that it is necessary to normalize the FIRM-PL data with respect to the power of FELIX at each given energy. In principle this can be achieved using one of three different methods. The first is to measure the initial FELIX power output within the beam line. The second is to measure the power density arriving directly at the cryostat windows. The third method is to use an internal calibration if a reference feature can be found. This third measure was chosen as the most accurate as it measures the

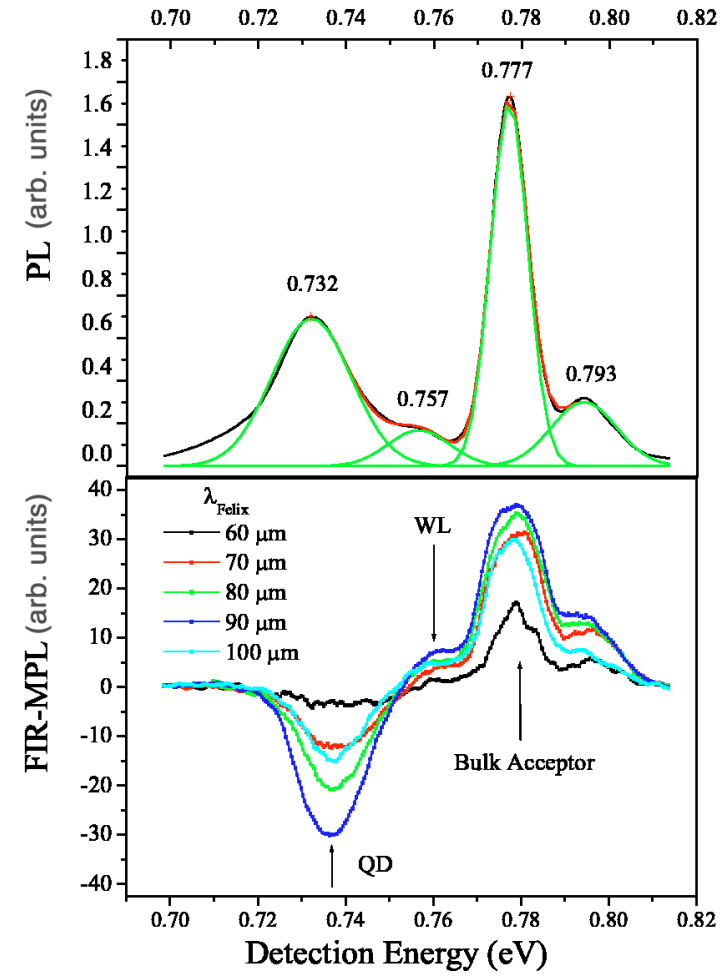

FIG. 3. Upper section shows photoluminescence (PL) data of sample $B$. The spectrum has not been normalized to take account of the spectral response of the Ge detector. The lower panel shows a comparison of the modulated PL signal for a series of different FIR wavelengths.

power density directly at the sample and should provide the most consistent method of calibration. This method could, however, only be used with sample $B$ due to the fact that it is grown on $\mathrm{GaSb}$ which results in enhanced signals from bulk $\mathrm{GaSb}$ features which can then be used for the normalization process.

Figure 3 shows the PL response of sample $B$. The quantum dot transition is at $0.73 \mathrm{eV}$ (Ref. 1) with the wetting layer (WL) the or bulk acceptor $B$ state being visible at 0.757 $\mathrm{eV}{ }^{12}$ There is a strong signal from the bulk acceptor $A$ state at $0.777 \mathrm{eV}$ which was used as the reference feature to measure the incident FIR power. ${ }^{12}$ As this state is sufficiently strongly bound not to show any resonant transitions in the FIR energy range used, it is expected that this feature can act as an accurate internal pyrometer. This assumption was checked by using an in-line power meter in the wavelength range $80-150 \mu \mathrm{m}$. A weaker peak at $0.793 \mathrm{eV}$ (BE4) originates from an acceptor bound exciton. ${ }^{12}$

The experiment was performed in the FIR high power limit in order to ensure sufficient signal at all wavelengths. Thus the effect of the FIR on the quantum dots was to reduce the overall level of the dot photoluminescence as indicated by a negative FIRM-PL peak, with a corresponding increase being observed in the wetting layer and bulk GaSb features. Figure 3 illustrates how the amplitude of the FIRM-PL signal of the quantum dot feature has a much stronger dependence on the FIR energy than that of the GaSb bulk features. It is therefore proposed that the FIR is inducing a resonant exci- 


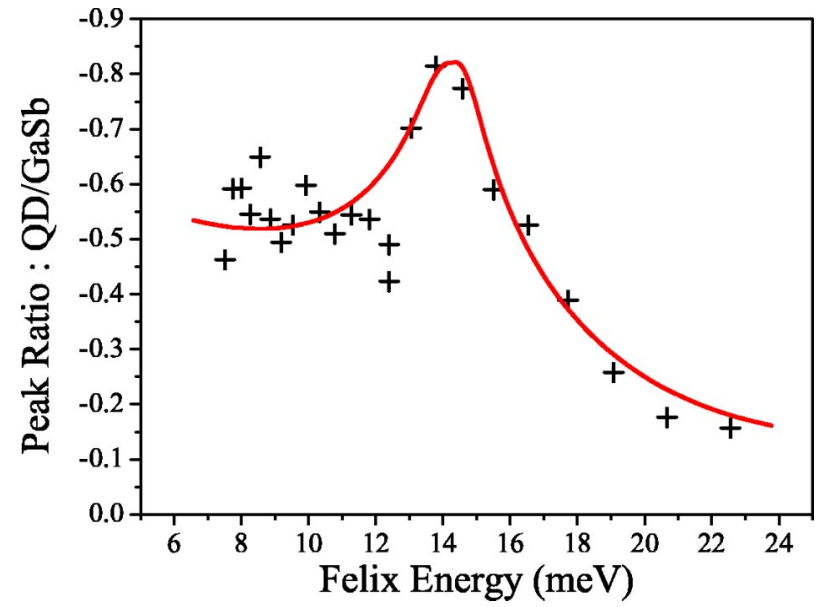

FIG. 4. Ratio of the amplitudes of the MPL features at the emission energies corresponding to the quantum dots and bulk GaSb.

tation in the quantum dots. The excited states in the dots lie much closer in energy to the WL and bulk GaSb and therefore have a higher probability of reionization before recombination occurs.

The resonant nature of the FIR response can be seen clearly when the normalized FIRM-PL response (the ratio of the quantum dot MPL peak to that of the bulk acceptor) is plotted in Fig. 4. There is a strong feature which peaks at $\sim 14.5 \mathrm{meV}$. We propose that this spectrum is a direct measure of the distribution of quantum dot excitation energies for occupied dots, measured in the high-temperature limit. For a simple harmonic confinement potential this will be the same as the distribution of dot confinement energies $\hbar \Omega_{d o t}$. Such a distribution is entirely consistent with the picture deduced by Alphandéry et $a .^{2}$ who demonstrated using magnetophotoluminescence experiments on $\mathrm{InSb} / \mathrm{GaSb}$ quantum dots, as a function of temperature and power, that a range of dots exist whose lateral confinement energies $\hbar \Omega_{d o t}$ vary significantly. ${ }^{2}$ In the low excitation power, low-temperature regime the average confinement energy of populated dots, $\hbar \Omega_{d o t}$, was found to be $\sim 6 \mathrm{meV}$. When significantly larger excitation laser powers were used, the mean energy of the occupied dots was found to shift significantly to higher energy with a corresponding increase in $\hbar \Omega_{d o t}$ up to $\sim 14 \mathrm{meV}$ being observed. ${ }^{2}$

An example of the excitation power dependence of the PL is shown for sample $B$ in Fig. 5, where a shift of $\sim 10 \mathrm{meV}$ in the dot transition energy with excitation power was observed. This would correspond to the difference between the preferential population at low temperature of large dots, and the uniform population of the quantum dot distribution brought about by a high temperature or high intensity. The peak observed at $14.5 \mathrm{meV}$ in Fig. 4 is consistent with the change in mean dot binding energies discussed above, while the width of the high intensity quantum dot PL in Fig. 5 is consistent with a range of confinement energies of order 20 $\mathrm{meV}$. The broad peak in the FIRM-PL is consistent with the fact that no higher energy peak, or excited states, has been resolved in PL for either high power interband excitation or with FIR modulation. The significant distribution of dot en-

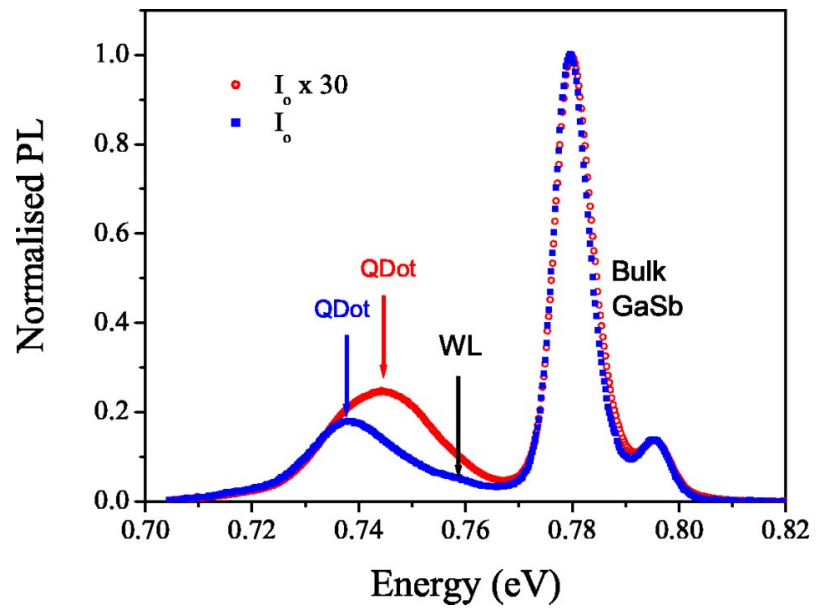

FIG. 5. The effect of the incident power on the PL of the quantum dots in sample $B$. Neutral density filters were used to attenuate the laser power.

ergies results in a substantial overlap of the excited states from larger dots with the ground state of smaller, highenergy dots thus smearing out the total PL signal.

The asymmetry of the spectrum in Fig. 4 is quite striking, with a rapid decrease in normalized FIRM-PL beyond 14-15 $\mathrm{meV}$ as the magnitude drops off to levels close to zero. This suggests that there must be a lower limit to the dot size. Taking the upper limit for the in-plane confinement energy to be $\sim 22 \mathrm{meV}$ from Fig. 4 and assuming an effective mass ${ }^{2}$ of $0.035 m_{o}$, the lateral extent of the peak in the distribution can be estimated as $\sim 25 \mathrm{~nm}$ and the smallest dots are $\sim 20 \mathrm{~nm}$. This is in good agreement with the estimates made in Ref. 2. A further contributing factor to the asymmetry of the distribution is that although we estimate the electron temperature to be of the order of $40 \mathrm{~K}$, this is not sufficiently high to cause a uniform distribution amongst the dots and will cause a significant preferential population of the lower confinement energy dots.

A further contributing factor to the falloff in response is likely to be the overlap of the excited states with the wetting layer. It is known in quantum wells that the strength of bound to continuum transitions decreases as the bound level approaches the barrier. ${ }^{13}$ The difference between the interband transition energies of the wetting layer and the (low intensity) dot ground state is of the order of $33 \mathrm{meV}$. As the distribution of band offsets in this system is approximately equal in the valence and conduction bands it is expected that the energy difference of their conduction-band energies would be of the order of $16 \mathrm{meV}$. For dots with excitation energies above this the excited state will be resonant with the wetting layer which will cause a further decrease in the transition probability due to the decreased overlap of the wave functions. The results of Sauvage et al. ${ }^{9}$ on photoinduced absorption indicate that for a sample with a broad distribution of ground-state energies, excited-state transitions dominate in the high-temperature limit and transitions to the wetting layer can only be detected in the low-temperature limit when the excited-state transitions are suppressed. The FIRM-PL measurements reported here are all performed in 
the high-temperature limit $(\mathrm{T} \gtrsim 10 \mathrm{~K})$, where ionization of the excited state is highly probable.

By contrast to the high-energy falloff, the quite extended tail of the absorption on the low-energy side of Fig. 4 suggests that there is a significant distribution of larger dots with very weak confinement energies. This observation is also consistent with the observations from the low intensity PL measurements ${ }^{2}$ that the dominant confinement energy is only $\sim 6 \mathrm{meV}$.

\section{SUMMARY AND CONCLUSION}

FIRM-PL measurements in InSb/GaSb quantum dots have been performed using a free-electron laser as the FIR source. The power density of the FIR on the sample was important and can be described by two regimes. In the low power limit the FIR modifies the nonthermal distribution of carriers in the dots by exciting transitions to excited states with a lower localization energy and allowing the population of large, low-energy dots. The second, high power limit results in a significant increase in the overall carrier temperature, with the electrons escaping from the large dots. A decrease in the dot photoluminescence was observed in this regime while the wetting layer FIRM signal increased.

The spectral dependence of the FIRM-PL signal was measured at high FIR powers and shows that there is an asymmetric distribution of occupied quantum dots with a peak excitation energy of around $14.5 \mathrm{meV}$.

\section{ACKNOWLEDGMENTS}

We gratefully acknowledge the support by the Stichting voor Fundamenteel Onderzoek der Materie (FOM) in providing the required beam time on FELIX and highly appreciate the skilful assistance by the FELIX staff. This work was supported in part under the "Access to research infrastructure action of the Improving Human Potential Program" of the European Community and by the UK-EPSRC.
${ }^{1}$ E. Alphandéry, R.J. Nicholas, N.J. Mason, B. Zhang, P. Möck, and G. Booker, Appl. Phys. Lett. 74, 2041 (1999).

${ }^{2}$ E. Alphandéry, R.J. Nicholas, N.J. Mason, S.G. Lyapin, and P.C. Klipstein, Phys. Rev. B 65, 115322 (2002).

${ }^{3}$ J.G. Michels, R.J. Warburton, R.J. Nicholas, and C.R. Stanley, Semicond. Sci. Technol. 9, 198 (1994).

${ }^{4}$ H. Drexler, D. Leonard, W. Hansen, J.P. Kotthaus, and P.M. Petroff, Phys. Rev. Lett. 73, 2252 (1994).

${ }^{5}$ M. Fricke, A. Lorke, J.P. Kotthaus, G. Medeiros-Ribeiro, and P.M. Petroff, Europhys. Lett. 36, 197 (1996).

${ }^{6}$ S. Sauvage, P. Boucaud, F.H. Julien, J.-M. Grard, and V. ThierryMieg, Appl. Phys. Lett. 71, 2785 (1997).

${ }^{7}$ J. Phillips, K. Kamath, and P. Bhattacharya, Appl. Phys. Lett. 72, 2020 (1998).

${ }^{8}$ R.J. Warburton, B.T. Miller, C.S. Dürr, C. Bödefeld, K. Karrai,
J.P. Kotthaus, G. Medeiros-Ribeiro, P.M. Petroff, and S. Huant, Phys. Rev. B 58, 16221 (1998).

${ }^{9}$ S. Sauvage, P. Boucaud, F.H. Julien, J.-M. Grard, and J.-Y. Marzin, J. Appl. Phys. 82, 3396 (1997).

${ }^{10}$ B.N. Murdin, A.R. Hollingworth, J.A. Barker, D.G. Clarke, P.C. Findlay, C.R. Pidgeon, J.-P.R. Wells, I.V. Bradley, S. Malik, and R. Murray, Phys. Rev. B 62, R7755 (2000).

${ }^{11}$ D. Bimberg, M. Grundman, and N. N. Ledentsov, Quantum Dot Heterostructures (Wiley, New York, 1999).

${ }^{12}$ E.T.R. Chidley, S.K. Haywood, A.B. Henriques, N.J. Mason, R.J. Nicholas, and P.J. Walker, Semicond. Sci. Technol. 6, 45 (1991).

${ }^{13} \mathrm{~B}$. Vinter and L. Thibeaudeau, in Intersubband Transitions in Quantum Wells, edited by E. Rosencher, B. Vinter, and B. Levine (Plenum, New York, 1992), p. 329. 